\title{
Secured and Reliable Urban Area Applications Based on IoT
}

\author{
Mayuri Khapne"1, Prof. Nekita A. Chavhan ${ }^{2}$ \\ ${ }^{1}$ M-Tech Scholar, CSE Department, G. H. Raisoni College of Engineering, Nagpur, Maharashtra, India \\ ${ }^{2}$ HOD, CSE Department, G. H. Raisoni College of Engineering, Nagpur, Maharashtra, India
}

\begin{abstract}
There is requisite and is iteratively expressed for a smart city kind development from different organisations in various countries around the world. Many countries are already looking forward to eliminate intricacies in different fields comprising those of industrial, technical and medicine by keeping a regular eye on as well as bringing a better kind communication. Perpetuating on cost and being reparatory on time makes to an exceptional level of progress. We can observe that automated type system is de facto taken over by the manual type system. Automation of home or any organisation utilising IoT is a system, which considers laptops or smart type phones primarily to superintendence the functions required in homes or even business premises. Appliances of house hold type, which in general comprises of television; lighting system and even doors and gates, and these, can be handled with a switch of a button. This is comprehended as centralised type and much security can be expected. Embedded kind technology chosen and worked herein helps in making to the best overall composition. Weather type conditions are imperative for monitoring primarily to keep goingness of a healthy growth of crop throughout the year. Due to a prelation of technology, environmental kind parameters have become plain sailing when compared to prior days. Superb kind sensors are worked out primarily to guesstimate measure and arrive with the physical and environment kind of parameters. In the successive pages, there is a discussion on how an IoT type platform is developed, various devices, which makes to overall composition. Various software and hardware systems are enlisted, which it is considered for the successful IoT type operation.
\end{abstract}

Keywords : Arduino, Wi-Fi (ESP 8266), Load cell, Database System

\section{INTRODUCTION}

Notion behind IoT as part of promoting to smart kind city makes or comprises of various newfangled services along with the rigorous utilisation of information and following to technology. Technologies behind smart type city constitute the usage of smart type phones, which are nowadays identified as the coalesce in the real world. In the broader sense, IoT and the cloud type computing can be indubitably recognised as the most significant among the ICT type models, which are in the point of fact, shaping the generation in next sequential years especially at computing. Hardware and also software essentials can be premeditated and made to deployment of applications specially developed for smart type cities. Healthcare type industries are the one to be got the most mileage. There are contemporary generation kind devices, which constitute devices like sensors, which are virtual as well as physical type and these are brought in link by an Internet means, especially for applications, which are value added type. In present times, crowd is considering shifting to developed kind cities for an 
enhanced type lifestyle hence naturally this concept becomes more demanding and less convoluted communication system.

Different department can work like knowledge sharing, considering digitalising, make to marketing and suggestion board for an incessant success in any future course of works. A city can be made in comparison to nervous kind organisation in human and communication, which can be expected likewise in flow. With repercussion from surge of Internet, information made for availability, has become rised herein. There is a need for well-regulated kind of interaction especially between the entities represented. An intelligent city herein reckon on digital type infrastructure, which actually interlinks between local type community and drives forth the growth, efficiency, furthering to productivity and even competitiveness. Role of the smart city kind managers need to be analysed and re-established.

\section{PROPOSED WORK}

Overall the goal of this work is to use a heterogeneous network of SOs using different communication technologies in order to perform real time traffic estimation in the cities, with the following principles like

(i)Sensing correctly the environment,

(ii)Exchanging the information securely

(iii)Safeguarding private information.

Users are reluctant to adopt this new technology that will be a part of their everyday lives, which decreases the market value of SC applications for the service providers. Therefore if these concerns are not addressed proactively at the early stages of IoT deployment for SCs they may act as a barrier to the adoption of this technology by users and businesses.
In this respect, the FP7 project RERUM aims to improve the IoT technology making it more reliable, trustworthy and secure for enabling both users and service providers to adopt it and enjoy its benefits.

\section{METHODOLOGY}

\section{PROPOSED AREAS OF APPLICATION:}

\section{TRAFFIC CONTROLLING (E.g.: AMBULANCE)}

\section{Existing System:}

\section{$>$ It is Difficult to identify the Traffic Violators.}

$>$ There is no IOT based Traffic management System.

\section{Proposed System:}

$>$ IOT based traffic management

$>$ Easy to find the path for emergency condition in ambulance.

$>$ The Traffic violators are captured and send to Police.

\section{Making way for Ambulance:}

RFID reader will be placed at the place such that it can detect the ambulance whenever it approaches the traffic around 100 meters near the traffic control system and will be connected to the controller. When the ambulance RFID tag will be read the RF transmitter will send signal to RF receiver which changes the traffic signal from red to green and makes way for the ambulance. 


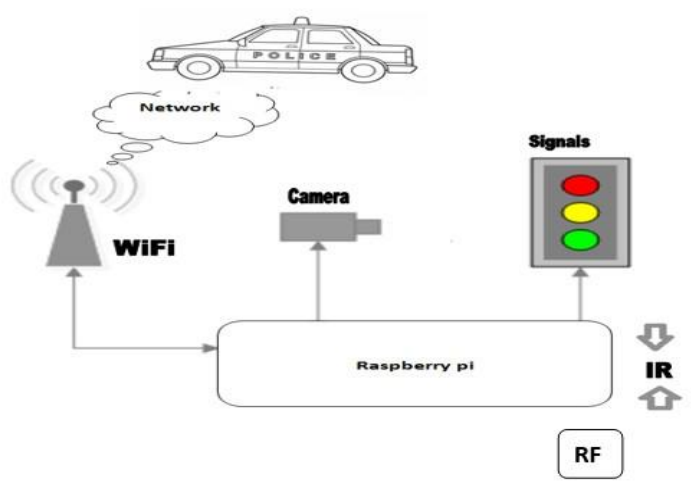

IV.LITERATURE REVIEW

To address the notion of Trust, RERUM will introduce the concept of trust in the core of the system and facilitate this through all its layers developing a cross-layer reputation management framework. The key concept is that using advanced fusion techniques only trusted SOs will be allowed to exchange data and malicious or misbehaving nodes sending false data will be excluded from the network. Thus, the reliability of the gathered data will be ensured. To measure the trustworthiness of SOs, a weight model capturing the data context will be used. Weight will not only determined by the input provided by users, but also by the time it was last updated and by the effect that the context really has in the related service.

Additionally, the reputation management framework will use advanced fusion techniques for the data gathered by all SOs for evaluating the results to identify malicious or misbehaving objects. Finally, the cryptographic mechanisms that RERUM will employ are also able to identify the source, integrity and accuracy of the information exchanged by the SOs.

\section{v. CONCLUSION}

This work discusses the visions of the newly launched FP7 project RERUM towards enhancing the reliability and the security of SC applications. This in return makes service providers also reluctant to investing in SC applications. RERUM has the citizen at the center of attention and by including city administrations in all phases of the project; it ensures that the citizens' requirements will drive the system design. Additionally, RERUM adopts the concept of "reliability, security and privacy by design" and employs a number of security, privacy and trust mechanisms, most of which will be embedded on the SOs to provide inherent security and reliability to the SC applications.

\section{REFERENCES}

[1]. A. Rial and G. Danezis, "Privacy-preserving smart metering," in Proceedings of the 10th annual ACM workshop on Privacy in the electronic society. ACM, 2011, pp. 49-60.

[2]. M. Jawurek, M. Johns, and F. Kerschbaum, "Plugin privacy for smart metering billing," in Privacy Enhancing Technologies. Springe,2011.

[3]. R. A. Hobday, "Indoor environmental quality in refurbishment," Historic Scotland Research Report 12, 2011.

[4]. N. Gazoni et al. "A framework for opportunistic routing in multi-hop wireless networks," in Proc of the 7th ACM PE-WASUN 2010.

[5]. E.Tragos et al., "The impact of interference on the performance of a multi-path metropolitan wireless mesh network," in ISCC, 2011.

[6]. T. Tryfonas, I. Sutherland, and I. Pompogiatzis, "Employing penetration testing as an audit methodology for the security review of voip: Tests and examples," Internet Research, vol. 17, no. 1, pp. 61-87, 2007.

Cite this article as : Mayuri Khapne, Prof. Nekita A. Chavhan, "Secured and Reliable Urban Area Applications Based on IoT", International Journal of Scientific Research in Science and Technology (IJSRST), Online ISSN : 2395-602X, Print ISSN : 2395-6011, Volume 6 Issue 2, pp. 701-703, March-April 2019. Available at doi : https://doi.org/10.32628/IJSRST1962156 Journal URL : http://ijsrst.com/IJSRST1962156 